Schwind / Böhm / Jehle (Fn. 26), § 155 Rn. 1; Müther (Fn. 1), 15.

6 Gusy / Lührmann, StV 2001, 49, 54; Kammeier (Fn. 1),

74 (m. w. Nachw.); Calliess / Müller-Dietz, § 155 Rn. 2.

7 BVerfGE 35, 202, 235; 98, 169, 200.

8 Kammeier (Fn. 1), 75; Calliess / Müller-Dietz, § 155 Rn. $6 \mathrm{f}$.

9 Kulas, in: Stober (Fn. 1), 35, $40 \mathrm{ff}$.

10 Bonk, JZ 2000, 435, 442; Ch. Wagner, ZRP 2000, 169, 172; Wohlgemuth, KrimPäd 2000, 89 f.

11 Vgl. Fn. 7.

12 Gusy / Lührmann, StV 2001, 49, 54; Calliess / MüllerDietz, § 155 Rn. 3, 5; Schwind / Böhm/Jehle, § 155 Rn. 1.

13 Calliess / Müller-Dietz, Einl Rn. 31, § 155 Rn. 5.

14 Arloth/Lückemann, § 155 Rn. 4; Calliess / MüllerDietz, $\S 155$ Rn. 4.

15 Arloth / Lückemann, § 155 Rn. 4.

16 Vgl. etwa Flügge / Maelicke / Preusker (Hrsg.), Das Gefängnis als lernende Organisation, 2001; Wischka u. a. (Fn. 1); Calliess / Müller-Dietz, Einl Rn. 45, § 155 Rn. 2. 17 Arloth/Lückemann, § 155 Rn. 2.

18 BVerfGE 40, 285. Vgl. Calliess / Müller-Dietz, Einl Rn. $34 \mathrm{f}$.

19 Gusy / Lührmann, StV 2001, 48, 54; Calliess / MüllerDietz, § 155 Rn. 9.
20 Calliess / Müller-Dietz, § 155 Rn. 7.

21 Calliess / Müller-Dietz, § 155 Rn. 9.

22 Calliess / Müller-Dietz, § 155 Rn. 10.

23 In der neuen Justizvollzugsanstalt Hünfeld obliegen dem privaten Unternehmen u. a. »die sozialarbeiterische, psychologische und pädagogische Betreuung der Gefangenen, die Beratungsleistungen für die Gefangenen (Drogen-, Ausländer-, Schuldnerberatung), die Organisation und Durchführung der Freizeitveranstaltungen für die Gefangenen, insbesondere der Gefangenensport « (Presseinformationen des Hessischen Ministeriums der Justiz vom 12.12.2004, 2. und 15.11.2005; Kirsch, KrimJ $2005,128,137 \mathrm{f}$.

24 So hat der private Unternehmer in der Justizvollzugsanstalt Hünfeld verschiedene Versorgungsleistungen etwa den Betrieb der Anstaltsküche und die Versorgung der Gefangenen mit Verpflegung - zu erbringen (vgl. Fn. 23).

25Calliess / Müller-Dietz, § 155 Rn. 3 a.E.

26 Calliess / Müller-Dietz, § 155 Rn. 11.

27 JVB-Presse, Nov. 2005, 2 ff., 3; Straubinger Tagblatt vom 13.10.2005.

28 H. Cornel, ZfStrVo 2005, 48; Müller-Dietz, ZfStrVo 2005, 38; ders., ZRP 2005, 156.
29 ZfStrVo 2005, 366. Über entsprechende Auswirkungen einer solchen Verfassungsänderung vgl. z. B. die Presseinformation vom 18.11.2005 aus dem Hamburger Senat, wonach Justizsenator Kusch »die Sicherheit als Vollzugsziel Nummer 1 1 gesetzlich festlegen will.

30 In diese Richtung geht z.B. die Kritik Käppners, SZ vom 8.11.2005, S. 4: »Im Strafvollzug etwa geistern abenteuerliche Privatisierungsideen herum, bei denen es vor allem ums Geld geht und weniger um das Recht. «

31 Vgl. z. B. Südwest-Presse vom 27.4.2005; Ruhrnachrichten vom 18.8.2005; NZZ vom 29 9.2005.

32 Vgl. z. B. Allgäuer Zeitung vom 30.4.2005; Badische Zeitung vom 26.9.2005; IHK Wirtschaft im Südwesten 11/2005, 42. Vgl. auch Fn. 16 und 31.

33 Z.B. Rifkin, Das Ende der Arbeit und ihre Zukunft, 1995; Beck, Die Zukunft von Arbeit und Demokratie, 1999.

34 Assheuer, Die Zeit v. 20.10.2005, 49.

35 FAZ vom 19.11.2005, S. 12.

36 Etscheit, Die Zeit vom 24.11.2005, S. 87.

\title{
Privatisierung von Strafvollstreckung am Beispiel der Vermeidung von Ersatzfreiheitsstrafen durch Gemeinnützige Arbeit in Bayern
}

Gabriele Kawamura-Reindl

W ährend die kontroverse Diskussion des Themas Privatisierung auch Einzug in die Strafjustiz gehalten hat gibt es in der Bundesrepublik seit Jahren viele konkrete Beispiele für eine Privatisierung von Maßnahmen der Strafvollstreckung durch Übertragung an Freie Träger: So ist zu denken an ambulante Maßnahmen nach dem JGG, deren Durchführung in großem Umfang durch Freie Träger der Jugendhilfe erfolgt, an den Täter-Opfer-Ausgleich, der auch im Bereich des Allgemeinen Strafrechts u. a. von Trägern der Freien Wohlfahrtspflege umsetzt wird, an professionelle Haftentlassenenhilfe in privater Trägerschaft, an die Gemeinnützige Arbeit zur Abwendung von Ersatzfreiheitsstrafen durch die Freie Straffälligenhilfe, an die Vereine Chance e.V. und Prisma e.V. in Baden-Württemberg, die Teile des Jugendstrafvollzuges übernehmen, an den österreichischen Verein Neustart e.V., dem in Teilen Baden-Württembergs Aufgaben der Bewährungshilfe übertragen wurden und die Übertragung des sog. Betreuungsmanagements auf einen privaten Träger in der teilprivatisierten hessischen JVA Hünfeld durch den britische Konzern Serco $\mathrm{GmbH}$, um nur einige zu nennen. Bei genauerer Betrachtung unterscheiden sich diese Privatisierungsmaßnahmen deutlich danach, wer Träger ist (Non-Profit vs. Profit-Unternehmen), wer sie iniitiert hat (Kriminalpolitik von unten vs. Beauftragung eines Privaten Trägers durch die Jus- tiz), welche Ziele sie verfolgen, welche konkreten Maßnahmen sie durchführen, wer die Kosten trägt und über welche (über) Handlungsspielräume sie verfügen, um nur einige Dimensionen zu nennen.

Privatisierung von Aufgaben Sozialer Arbeit im Rahmen der Strafvollstreckung ist also auch in Deutschland nicht neu, sondern vielmehr eine Art von Sammelkategorie für das Outscourcing von Maßnahmen, die die Justiz anordnet, die aber von privaten Trägern umgesetzt werden. Die zunehmende Finanzknappheit der öffentlichen Hand hat dazu geführt, dass die Grundsätze der Wirtschaftlichkeit und Sparsamkeit zunehmend zu einer Prüfung der Frage führen, inwieweit staatliche Aufgaben durch Ausgliederung und Entstaatlichung oder Privatisierung erfüllt werden können. In vielen Bereichen öffentlicher Verwaltung wird zur Konkretisierung dieser Privatisierungsmaßnahmen zunehmend der Begriff »Public Private Partnership benutzt. »Public Private Partnership (PPP) bezeichnet das partnerschaftliche Zusammenwirken von öffentlicher Hand und Privatwirtschaft mit dem Ziel einer besseren wirtschaftlichen Erfüllung öffentlicher Aufgaben als bisher. PPP-Projekte erfassen das gesamte Spektrum zwischen der rein hoheitlichen Realisierung öffentlicher Aufgaben einerseits und der vollständigen Privatisierung öffentlicher Aufgaben andererseits. « (Bertelsmann Stif- tung u.a. 2005). Unter den Oberbegriff PPP lassen sich unterschiedlichste Sachverhalte und Erscheinungsformen subsumieren, die von formaler Privatisierung (= Organisationsprivatisierung), Teilprivatisierung, Vollprivatisierung, materieller Privatisierung (= Aufgabenprivatisierung) bis zur funktionalen Privatisierung (= Contracting out) reichen. Bei der Privatisierung von Aufgaben Sozialer Arbeit im Rahmen der Strafvollstreckung dürften wir es v. a. mit letzteren beiden Formen zu tun haben. In den meisten Fällen liegt dem $\mathrm{Zu}$ sammenwirken zwischen öffentlicher Verwaltung und privaten Trägern in der Regel eine vertragliche Regelung zugrunde, die gegenseitige Rechte und Pflichten, Laufzeiten, Leistungen, Vergütungen und andere Konditionen festschreibt. Hiervon ist die Straffälligenhilfe in einigen Bereichen der Privatisierung, z. B. dem Täter-Opfer-Ausgleich oder der Strafentlassenenhilfe noch relativ weit entfernt. Dies betrifft v. a. die Laufzeiten (eine auf ein Jahr festgelegte und damit ungesicherte Finanzierung darf inzwischen als die Regel und nicht als die Ausnahme betrachtet werden und stellt keine tragfähige Basis für längerfristige Planungen dar), aber auch die vertragliche Festschreibung von Leistungen und Vergütungen. Nicht nur in der Straffälligenhilfe verlagert der Staat durch Privatisierungen finanzielle Risiken auf private Träger, die bei einer absehbaren Deckelung zuvor budgetierter 
Kosten und vereinbarten Leistungsverträge kaum eine andere Möglichkeit haben, als die Belastungen den Mitarbeitenden in Form von arbeitsrechtlichen Restriktionen aufzubürden.

Die Vermittlung in Gemeinnützige Arbeit bei uneinbringlichen Geldstrafen zur Abwendung von Ersatzfreiheitsstrafen in Bayern wurde in den letzten Jahren weitgehend privatisiert - diese Entwicklung soll einer kritischen Beleuchtung unterzogen sollen. Am 6. April 2000 veranstaltete der Fachbereich Sozialwesen der Georg-Simon-Ohm-Fachhochschule Nürnberg gemeinsam mit dem Nürnberger Arbeitskreis Straffälligenhilfe eine landesweite Fachtagung »Gemeinnützige Arbeit in Bayern am Schnittpunkt von Sozialer Arbeit und Justiz Fakten - Erfahrungen - Lösungen «, mit der die Veranstalter eine qualitative und quantitative Bestandsaufnahme der Gemeinnützigen Arbeit erheben und gemeinsam mit der bayerischen Justiz kriminalpolitische Perspektiven entwickeln und ausloten wollten. Dieser Vorstoß wurde von Seiten der bayerischen Justiz ermutigt, die bis dahin das sog. Rechtspflegermodell, also die Vermittlung gemeinnütziger Arbeit bei uneinbringlichen Geldstrafen durch die Rechtspfleger bei den Staatsanwaltschaften favorisiert hatte. In seinem Geleitwort betonte der damalige bayerische Justizminister: »Die weitere Intensivierung der gemeinnützigen Arbeit zur Vermeidung der Ersatzfreiheitsstrafe ist seit Beginn des Modellprojekts ,Schwitzen statt Sitzen' Anfang der 80-er Jahre ein sehr wichtiges rechtspolitisches Anliegen des Bayerischen Staatsministeriums der Justiz. Die Vermeidung der Ersatzfreiheitsstrafe durch die Leistung gemeinnütziger Arbeit ist dabei nicht nur unter dem Gesichtspunkt der Einsparung von Kosten zu sehen. Bedeutsam ist vor allem auch unter dem Aspekt, daß das Gericht, eine Freiheitsstrafe im konkreten Fall gerade nicht für erforderlich gehalten hat. Die Freiheitsentziehung ist im Gesetz lediglich deshalb als Ersatzsanktion vorgesehen, weil die Verurteilten, bei denen sich die verhängte Geldstrafe nicht beibringen läßt, nicht straflos davonkommen können.«(Weiß 2000, S. 5). Nachdem gemeinnützige Arbeit in Bayern lange im Wege des sog. Rechtspflegermodells vermittelt wurde sah man sich mit dem Problem geringer Vermittlungsquoten konfrontiert: »Ein erhebliches Problem ist es aber nach wie vor, die für die Leistung gemeinnütziger Arbeit in Betracht kommenden Verurteilten für diese Möglichkeit zu motivieren. So haben im Jahr 1999 über 14.500 Verurteilte von den Vollstreckungsbehörden einen Hinweis auf die Möglichkeit, die Geldstrafe durch gemeinnützige Arbeit zu tilgen, erhalten. Lediglich 1.760 Verurteilte haben das Angebot überhaupt angenommen.«(ebd, S. 5 )

Bestätigt wurden diese Schwierigkeiten durch Erfahrungen einiger freier Träger der Straffälligenhilfe, die nach ersten Erfahrungen $u$. a. darauf verwiesen, dass Motivationsarbeit mit den häufig sehr problembelasteten Verurteilten sowie die Passgenauigkeit zwischen Verurteiltem und Einsatzstelle zwei sehr bedeutsame Kriterien für die erfolgreiche
Durchführung gemeinnütziger Arbeit zur Abwendung von Ersatzfreiheitsstrafen darstellen. Dass diese Aufgaben von den Rechtspflegern neben ihren sonstigen Aufgaben kaum erfolgreich zu leisten sind, bestätigen auch die Ergebnisse schon länger zurück liegender Untersuchungen (vgl. Jehle u.a. 1990; Kähler 2002). Ein weiteres, erhebliches Problem stellte die Finanzierung dar. Zwar führten schon einige Träger in Bayern gemeinnützige Arbeit durch, aber aus dem Landeshaushalt standen keine Mittel zur Finanzierung dieser Maßnahme zur Verfügung. Die freien Träger, die 2001 schon gemeinnützige Arbeit vermittelten, gerieten an die Grenzen dessen, was ihre Trägervereine finanzieren konnten und wollten eine verbindliche finanzielle Beteiligung des Landes, zumal es sich um eine Aufgabe im Rahmen der Strafvollstreckung handelte und die Justiz durch die gemeinnützige Arbeit bereits damals durch vermiedene Hafttage Einsparungen verzeichnen konnte. All dies war jedoch zahlenmäßig kaum dokumentiert und bot daher wenig Möglichkeiten, der bayerischen Justiz als starker und verlässlicher Verhandlungspartner gegenüber zu treten. Folgerichtig musste ein Zusammenschluss her, der Daten sammelte, Verfahrensweisen plante und der Justiz als Kooperationspartner mit einer Stimme gegenüber treten konnte. 2003 begründeten auf Initiative der Geschäftsführer des Treffpunkt e.V. in Nürnberg und der Münchner Zentralstelle für Strafentlassenenhilfe (MZS) die bayerischen Fachstellen zur Vermittlung gemeinnütziger Arbeit auf Landesebene eine Arbeitsgemeinschaft der bayerischen Vermittlungsstellen für gemeinnützige Arbeit (AG V). Mitglieder sind inzwischen die Brücke Aschaffenburg, die Fähre Bayreuth, die MZS München, der Treffpunkt e.V. Nürnberg, die AGS Würzburg, Die Gruppe Hof, Lifeline Bamberg, der SKF München und der Kontakt Regensburg und der evangelische Beratungsdienst der Inneren Mission München. Die AG V einigte sich zunächst einmal auf eine einheitliche Zählweise für die bearbeiteten Fälle und sammelte Daten aus allen beteiligten Fachstellen. Bereits anhand der Daten für das Jahr 2003 zeigte sich, dass die Einsparung von Hafttagen durch die von den Fachstellen erfolgreich bearbeiteten Fälle ein deutlich größeres Ausmaß hatte als die bis vor einigen Jahren von den Rechtspflegern der Justiz selbst durchgeführte Vermittlung. Bereits 2003 vermieden die bayerischen Mitgliedseinrichtungen der AG V bei den uneinbringlichen Geldstrafen 30.732 Hafttage, was einer jährlichen Kapazität von 84 Haftplätzen entsprach.

Die Chance, angesichts dieser Zahlen mit der Justiz in Verhandlungen über die Finanzierung einzutreten, nutzte die AG V mit dem erfreulichen Ergebnis, dass es inzwischen zu einer Vereinbarung zwischen den Einsatzstellen und dem Bayerischen Staatsministerium der Justiz gekommen ist. Diese Vereinbarungen mit den Einsatzstellen beinhalten, dass den Einrichtungen für jeden erfolgreich abgewendeten Tag Freiheitsstrafe eine Zuwendung gewährt werden kann, auf die die Einrichtungen allerdings keinen Rechtsanspruch erheben können.
Die Höhe dieser Zuwendung bestimmt sich nach den beiden Haushaltstiteln, die v. a. die Verpflegung und die soziale Betreuung beinhaltet, geteilt durch die Anzahl der insgesamt angefallenen Hafttage des Vorjahres. Für das Jahr 2005 hat das Bayerische Staatsministerium der Justiz die Zuwendung auf $4,71 €$ pro vermiedenem Hafttag festgesetzt. Dem zunächst einmal erfreulichen Umstand, dass die Justiz sich an den Kosten für die gemeinnützige Arbeit beteiligt und durch nicht vollstreckte Ersatzfreiheitsstrafen im Vollzug eingesparte Mittel zugunsten einer ambulanten Sanktion umverteilt steht das Problem entgegen, dass mit einer Zuwendung von etwa 4 bis $5 €$ natürlich die bei der Vermittlung gemeinnütziger Arbeit entstehenden Vermittlungs- und Betreuungskosten nicht annähernd gedeckt werden können, denn: sicher gibt es den einen oder anderen Geldstrafenschuldner, bei dem ein einziges Vermittlungsgespräch eine reibungslose Ableistung der gemeinnützigen Arbeit nach sich zieht, aber diese Geldstrafenschuldner stellen nach den Erfahrungen der Projekte und nach dem, was aus Untersuchungen über die soziale Situation der Klientel bekannt ist, die Minderheit dar. Für gemeinnützige Arbeit kommen nach den derzeit geltenden rechtlichen Bestimmungen die Personen in Betracht, bei denen seitens der Staatsanwaltschaft die Uneinbringlichkeit der Geldstrafe festgestellt wird, d. h. die Personen, die ansonsten von der Verbüßung eine Ersatzfreiheitsstrafe bedroht wären. In den vergangenen zehn Jahren gab es eine Reihe von Untersuchungen, die die soziale Situation von Geldstrafenschuldnern beleuchteten und deren Ergebnisse in einem Gutachten der Kriminologischen Zentralstelle Wiesbaden (Sohn 1999) zusammengefasst wurden. Bei Gefangenen, die Ersatzfreiheitsstrafen verbüßen handelt es sich überwiegend um sozial randständige Deutsche oder in Deutschland nicht integrierte Ausländer. Vorherrschende Problemlagen sind soziale Desintegration in Form von Arbeitslosigkeit, Armut, Wohnungslosigkeit, Abhängigkeit von legalen und illegalen Drogen und mangelnden tragfähigen soziale Kontakte. Diese Klientel benötigt ein deutlich höheres $\mathrm{Maß}$ an sozialer Betreuung als mit einem kurzen Beratungsgespräch zu erledigen wäre. Verfügten die Klienten über das nötige Ausmaß an Selbständigkeit und Kompetenz im Umgang mit Behörden, so wäre das in einigen Bundesländern übliche Rechtspflegermodell erfolgreicher gewesen. Die Zahlungsunfähigkeit der Betroffenen resultiert nach Erfahrungen der Praxis der Vermittlungsstellen in der Regel aus einer oder mehreren sozialen/materiellen Problemlagen, die in unterschiedlichen $\mathrm{Ab}$ stufungen wirksam werden: Dies sind kurzfristig durch Arbeitslosigkeit in materielle Not geratene Verurteilte, langfristig und schwer vermittelbare Arbeitslose und/oder Sozialhilfeempfänger, allein erziehende Elternteile, Drogen- bzw. Alkoholabhängige, Rentner, Psychiatriepatientinnen und patienten und/oder strafrechtlich vorbelastete Personen. Für diese Gruppen treffen Gemeinsamkeiten und Problemkumulationen zu, die in der Betreuungsarbeit Berücksichtigung finden müssen, und die die Ableistung gemeinnütziger Arbeit deutlich 
beeinflussen bzw. beeinträchtigen können. Hierzu zählen nach den Erfahrungen von Einrichtungen: materielle Existenzprobleme, familiäre Probleme, Auflösung tragfähiger sozialer Kontakte, berufliche Dequalifizierung, Mangel an sekundärer Arbeitsfähigkeit (Zeitstruktur- und Regelverlust), Wohnungsprobleme bis hin zur Wohnungslosigkeit, hohe Verschuldung, bedingte Arbeitsunfähigkeit durch Berufskrankheiten oder auch Krankheiten anderer Art, Alkohol- und Drogenprobleme, Lebens- und Versagensängste. Übereinstimmend berichten die Vermittlungsstellen, dass besonders der Arbeitseinsatz von Drogenabhängigen mit extremen Problemen verbunden ist und sich vor allem aufgrund der (krankheitsbedingten) Diskontinuität nur sehr wenige Einsatzstellen als geeignet erweisen, diese Klientel aufzunehmen.

Eine soziale Betreuung am Rande der Vermittlung begründet sich vor allem mit häufig vorliegenden Mehrfachproblembelastungen der Klientel, die Beratung und Kriseninterventionen (z. B. vermittelnde Interventionen zwischen Verurteilten und Arbeitsstelle bei auftretenden Konflikten) erforderlich machen - nicht nur, um die gemeinnützige Arbeit erfolgreich abzuwickeln, sondern auch, um weitere Notlagen abzuwenden und eine Stabilisierung der Lebenssituation der Betroffenen zu erreichen.

Bezogen auf die Kooperation sind Zuverlässigkeit und Transparenz wichtige Qualitätsmerkmale sozialer Dienstleistungen. Von der Genehmigung zur Ableistung der Gemeinnützigen Arbeit bis hin zu deren Erledigung sind eine Vielzahl von Personen und Stellen involviert: Die Rechtspfleger bei den Staatsanwaltschaften, der Schuldner/Verurteilte, die Fachstelle, die Einsatzstelle und evtl. andere Behörden und Stellen. Damit ist der ganze Prozess der Vermittlung gemeinnütziger Arbeit von vielen Schnittstellen geprägt (vgl. KawamuraReindl / Reindl 2003). Mangelnde Informationen, unterschiedliche Kommunikation oder unklare Abläufe und Vereinbarungen können das Fehlerrisiko deutlich erhöhen und/oder zu Ungleichbehandlungen führen. Deshalb wurden 2005 von den Mitgliedern der bayerischen AG V gemeinsame Standards für die Vermittlungsstellen entwickelt und festgelegt, die im Frühjahr 2006 unter dem Titel "Ziele und Leistungen der Fachstellen zur Vermittlung gemeinnütziger Arbeit in Bayern « veröffentlicht werden und die eine gemeinsame fachliche, standardisierte Grundlage für die weitere Vermittlungstätigkeit freier Träger in Bayern bilden. Das standardisierte Verfahren garantiert allen Beteiligten eine hohe Verbindlichkeit der Absprachen, eine bessere Kommunikationsstruktur, die Gleichbehandlung der Geldstrafenschuldner, straffe, zeitliche Abläufe und ein gleiches Maß an Kontrolle. In der Fachstelle für gemeinnützige Arbeit (FaGA) des Treffpunkt e.V. Nürnberg wurde darüber hinaus vor kurzem eine Zufriedenheitsbefragung der Einsatzstellen durchgeführt, die derzeit in Zusammenarbeit mit der Verfasserin ausgewertet wird. Auch diese Ergebnisse werden zur
Qualitätssicherung, -verbesserung und -weiterentwicklung beitragen.

Die bisherigen Erfolge dieser Strafvollstreckungs-Privatisierung lassen sich sehen: Die inzwischen recht etablierten bayerischen Fachstellen zur Vermittlung gemeinnütziger Arbeit sind fachlich gut präpariert, um auch weiterhin dem Vermittlungs- und Hilfebedarf der Klienten wie der beteiligten Institutionen Rechnung zu tragen. Die Ziele der Sozialarbeit bei der Vermittlung gemeinnütziger Arbeit zur Abwendung von Ersatzfreiheitsstrafen (vgl. Cornel 2002, S. 828), also die Vermeidung von Prisonisierungsschäden und die Verhinderung einer zusätzlichen Benachteiligung ohnehin vermögens- und beziehungsloser Menschen durch Vollstreckung von Ersatzfreiheitsstrafen werden weitgehend erreicht. Die erfolgreiche Vermittlung gemeinnütziger Arbeit bei den uneinbringlichen Geldstrafen dürfte auch die Angehörigen von zu Geldstrafen Verurteilten entlasten, die früher häufig unter großen Schwierigkeiten und unter Inkaufnahme weiterer Schulden den Verwandten in der Haftanstalt »auslösten «, um ihn vor den Belastungen des Freiheitsentzugs zu schonen. Die Vermittlungsstellen verfügen inzwischen über ein breites Spektrum an Einsatzstellen und eine etablierte Kooperation mit den verschiedenen Stellen - die befürchteten Einbrüche auf dem Markt der »Freien Arbeit« durch die sog. 1-EuroJobs blieben bislang aus. Die erste vertragliche Vereinbarung mit dem bayerischen Staatsministerium der Justiz war ein wichtiger Meilenstein zu einer dauerhaften, verlässlichen Public Private Partnership. Die Anzahl der von den AG V-Mitgliedsorganisationen 2004 bearbeiteten uneinbringlichen Geldstrafen (357.866 abgeleistete Stunden = 62.108 eingesparte Hafttage) hat sich gegenüber dem Vorjahr mehr als verdoppelt und bedeutet für die bayerische Justiz inzwischen eine Einsparung von etwa 170 Haftplätzen pro Jahr. Die Arbeit der Projekte leistet damit nicht nur einen Beitrag zur Kosteneinsparung auf Seiten der Justiz, sondern angesichts sozialer Polarisierungen und sich verschärfender materieller Notlagen von straffällig Gewordenen auch zum Umbau stationärer zugunsten ambulanter Sanktionsmaßnahmen.

Gleichwohl bleibt noch einiges zu tun: Mit dem derzeit von der Justiz übernommenen Kostenbeitrag können die Einrichtungen nicht annähernd kostendeckend arbeiten, d. h. ohne eine deutliche Aufstockung der justitiellen » Tagessatzes « oder weitere Zuweisungen von Geldbußen kann die Arbeitsfähigkeit der Projekte nicht erhalten bleiben. Wünschenswert wäre ferner eine stärkere Verbindlichkeit dieser vertraglichen Vereinbarung seitens der Justiz, die den Trägern mehr personelle Planungssicherheit ermöglicht, denn die Erfolge solcher Maßnahmen - dies zeigt der steigende Anteil der vermittelten Arbeitsstunden - steht und fällt mit der Kontinuität und Erfahrung engagierter Mitarbeiterinnen und Mitarbeiter.
Gerade die Tatsache, dass in Bayern die Gemeinnützige Arbeit zur Abwendung von Ersatzfreiheitsstrafen nicht dem Profit-, sondern dem Non-Profit-Sektor übertragen wurde, bietet im Kontext sich entwickelnder Standards eine deutlich geringere Gefahr von Creaming-Effekten. Gemeint ist damit also die Ausgrenzung besonders problembelasteter Klientel zugunsten einfach vermittelbarer Verurteilten unter erfolgs- und damit gewinnorientierten Gesichtspunkten. Hier verfügen sozialpädagogische Non-Profit-Unternehmen nicht nur über eine ethische Grundhaltung, die solchen Ausgrunzungen entgegenwirkt, sondern eben auch über rechtliche Möglichkeiten, etwa durch Bußgeldeinwerbungen wenigstens zeitweilig finanzielle Engpässe zu kompensieren. Eine unter Kostengesichtspunkten reduzierte Dienstleistung durch die Mitarbeiter der Vermittlungsstellen würde den schwierigen Lebenslagen der Klientel nicht mehr gerecht, würde problemlosere Klientel bevorzugen und letztlich auch die Erfolge bei der Vermittlung gemeinnütziger Arbeit deutlich reduzieren. Das Argument der Justiz, es sei nicht ihre Aufgabe, sich über die Reduzierung von Haftkosten hinaus auch um die soziale Situation und Betreuung der Geldstrafenschuldner zu kümmern, greift somit zu kurz, denn nur die Betreuung, die passgenaue Vermittlung und die nötige Krisenintervention sichern letztlich hohe Erfolgsquoten und damit auch Einsparungseffekte auf Seiten der Justiz. Für dauerhaft unzureichende Finanzierung der Vermittlungstätigkeit der Freien Träger wird also auch ein Preis zu zahlen sein.

Derzeit verzeichnen die bayerischen Vermittlungsstellen angesichts ihrer erfolgreichen Arbeit einen Wunsch der Amtsgerichte nach vermehrter Vermittlung und Begleitung der Bewährungsauflagen ohne Bewährungshelfer bei Erwachsenen. Diese Leistungen finanzieren die meisten Vermittlungsstelle derzeit ausschließlich durch Bußgeldzuweisungen - auch hier wäre eine tragfähigere Regelung sinnvoll. Gleichzeitig wäre allerdings nicht ausschließlich, aber ganz besonders beim Einsatz gemeinnütziger Arbeit als Bewährungsauflage eine kritische Beobachtung möglicher NetWidening-Effekte, wie sie für die gemeinnützige Arbeit schon in mehreren ausländischen Untersuchungen bereits nachgewiesen worden sind (vgl. Schneider 2001, S. 279 ff.), angebracht. Denn solche Ausweitungen strafrechtlicher Kontrolle (also etwa zusätzliche Bewährungsauflagen) sind nicht nur kriminalpolitisch wenig wünschenswert, sondern sie könnten auch die Kosteneffektivität der gemeinnützigen Arbeit gegenüber dem Vollzug mindern.

Eine Privatisierung von Teilen der Strafvollstreckung ist also nicht per se gut oder schlecht - ihre Vor- und Nachteile hängen ab von den Zielen, die man sich setzt, von der Ausstattung und der vertraglichen Gestaltung der Zusammenarbeit, von der Art der privaten Unternehmen, die man beauftragt und von den auftretenden Nebenwirkun- 
gen. Der Anreiz zu solchen öffentlich-privaten Partnerschaften ergibt sich nicht einseitig durch einen der beiden Partner, sondern gerade durch die spezielle Ausrichtung und Gestaltung einer langfristigen, fairen und vertraglich fixierten Partnerschaft beider, bei der neue und Erfolg versprechende Wege einer effizienteren Zusammenarbeit zwischen öffentlicher Hand und Privatsektor genutzt werden - in diesem Fall nicht nur zur Kosteneinsparung, sondern für etwas Besseres als den Freiheitsentzug.

\section{Literatur:}

Bertelsmann Stiftung u.a. (Hg.): Prozessleitfaden Public Private Partnership, URL: http://www.initiatived21.de/ themen/egovernment_pppleitfaden/doc/16_10577646 82.pdf vom 19.12.05

Cornel, Heinz: Gemeinnützige Arbeit zur Abwendung der Vollstreckung von Ersatzfreiheitsstrafen und als selb- ständige Sanktion, in: Prittwitz, Cornelius u. a. (Hg.): Festschrift für Klaus Lüderssen, Baden-Baden 2002, S. 821-834

Jehle, Jörg Martin; Feuerhelm, Wolfgang; Block, Petra: Gemeinnützige Arbeit statt Ersatz-freiheitsstrafe - Berichte, Materialien, Arbeitspapiere, Heft 4, Kriminologische Zentralstelle, Wiesbaden 1990

Kähler, Anja: Tilgung uneinbringlicher Geldstrafen durch gemeinnützige Arbeit. Praktische Möglichkeiten der Haftvermeidung - untersucht und erörtert am Beispiel des Praxisprojektes Gemeinnützige Arbeit beim Caritasverband Geldern-Kaevelaer e.V.. Münster: LIT 2003

Keicher, Rolf; Anhorn, Roland: Privatisierung als Chance? Straffälligenhilfe zwischen marktwirtschaftlicher und staatlicher Steuerung. Freiburg: Lambertus 2005

Kawamura, Gabriele: Gemeinnützige Arbeit in Bayern am Schnittpunkt von Sozialer Arbeit und Justiz. Fakten Erfahrungen - Lösungen. Nürnberg: Georg-SimonOhm-Fachhochschule 2000

Kawamura-Reindl, Gabriele; Reindl, Richard: Rahmenbedingungen erfolgreicher Ableistung gemeinnütziger Arbeit: Evaluationsergebnisse des Modells der Fachstel- len in Nordrhein-Westfalen, in Neue Kriminalpolitik, 15. Jg., H. 2/2003, S.49-52

Schneider, Ursula: Gemeinnützige Arbeit als Zwischensanktion, in Monatsschrift für Kriminologie und Strafrechtsreform, 84. Jg., H. 4/2001, S. 273-287

Sohn, Werner (1999). Gutachten der Kriminologischen Zentralstelle zur kurzen Freiheitsstrafe und zur Ersatzfreiheitsstrafe im Auftrag des Bundesministeriums der Justiz. Bearbeitungszeitraum: 1. Mai - 1. August 1999. Wiesbaden: KrimZ

Weiß, Manfred: Geleitwort des bayerischen Staatsministers der Justiz, in Kawamura 2000, S. 5

Gabriele Kawamura-Reindl ist Kriminologin und Sozialarbeiterin, Professorin am Fachbereich Sozialwesen der Georg-Simon-OhmFachhochschule Nürnberg und Mitherausgeberin der NK.

\section{Auf dem Weg zu einem europäischen Modell von Bewährungshilfe?}

m Frühjahr 2004 veröffentlichte das Justizministerium Baden-Württemberg in diversen Printmedien Europas eine »Beschränkte Ausschreibung mit öffentlichem Teilnahmewettbewerb «. Die Leistung, die von den Anbietern erwartet wurde, war nichts weniger als die Erledigung der Aufgaben der Bewährungs- und Gerichtshilfe in zwei Gerichtbezirken im Rahmen eines zeitlich auf zwei Jahre beschränkten Pilotverfahrens. Im Anschluss an diese Pilotphase ist die flächendeckende Aufgabenübertragung im gesamten Land BadenWürttemberg vorgesehen. Aufgabe des Anbieters war die Erstellung eines fachlichen und organisatorischen Konzepts für die Durchführung der Pilotierung, wobei größtmögliche Effizienz und Effektivität gewährleistet und nach einheitlichen und zweckmäßigen Standards sichergestellt sein soll. Weiter muss die Übertragbarkeit der organisatorischen und fachlichen Konzeption auf die übrigen Landgerichtsbezirke gesichert sein.

Diese Entscheidung löste heftige fachpolitische Kontroversen aus, deren Ton sich naturgemäß verschärfte, als im Oktober 2004 das Justizministerium den Zuschlag für diesen Auftrag nicht dem favorisierten eigens zu diesem Zweck von den Landesverbänden der Bewährungshilfe gegründeten lokalen Sozialunternehmen, sondern von sieben Bewerbern dem österreichischen Unternehmen »Neustart « erteilt wurde. Ausschlaggebend für diese Entscheidung war laut Pressemitteilungen u. a. dass »Neustart« als Unternehmen langjährige
Erfahrung mit der Durchführung von sozialarbeiterischen Leistungen für die Justiz aufweist, über eine übertragbare Organisationsstruktur sowie über ein Netz von ehrenamtlichen Bewährungshelfern verfügt und ein überzeugendes Projektkonzept vorlegte.

Nach der Zuschlagserteilung wurden zwischen dem Justizministerium und dem Sozialunternehmen »Neustart« weitere Details ausverhandelt und vertraglich festgelegt. Die einzelnen Ziele wurden in einem Projektplan festgelegt und das begleitende Projektcontrolling einem gemeinsam besetzten Lenkungsausschuss übertragen. Eine wissenschaftliche Evaluierung konnte wegen fehlender Finanzierbarkeit nicht vergeben werden. Seit 1.1.2005 wird das Projekt umgesetzt und verläuft laut Aussage des zuständigen Beamten des Justizministeriums Michael Steindorfer außerordentlich zufriedenstellend. U.a. wurden bereits 65 Personen gefunden, die bereit und fähig sind, als ehrenamtliche Bewährungshelfer selbständig und eigenverantwortlich Betreuungsarbeit zu leisten, womit in einem zentralen Bereich des Projekts die Zielgröße von 90 bis 110 nahezu erreicht wurde. Die Wogen haben sich weitgehend geglättet, was auch auf die gelungene Informationspolitik des Sozialunternehmens in den Medien und innerhalb der Fachöffentlichkeit zurückzuführen ist. Dementsprechend glücklich ist man auch in der Wiener Zentrale von »Neustart « und bereitet sich laut Auskunft des für die organisatorische
Durchführung verantwortlichen Geschäftführers Wolfgang Herrmann bereits auf den nach EURichtlinien vorgesehenen »Wettbewerblichen Dia-

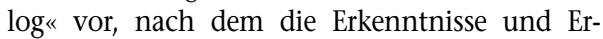
gebnisse des Pilotversuchs in allen Gerichtsbezirken Baden-Württembergs implementiert werden sollen. Diese Entwicklung entspricht dem Leitbild von »Neustart", der sich auch als europaweit tätige Expertenorganisation in Sachen Bewährungshilfe und anderen persönliche Dienstleistungen in der Justiz etablieren will.

Nach den mir vorliegenden Unterlagen handelt es sich hier um ein sehr gelungenes Beispiel einer Privatisierung und man kann den Projektverantwortlichen dazu nur gratulieren. Ich halte das Projekt für einen wichtigen Beitrag europäischer Integrationsbemühungen im Bereich des Sozialen, bei der die Qualität der Leistungserbringung für die KlientInnen im großen und ganzen verbessert werden konnte und weitere Qualitätsverbesserungen erwartet werden können. Das liegt auch in der humanistischen Tradition des Vereins »Neustart « und der dort entwickelten sozialarbeiterischen und organisatorischen Kompetenzen begründet. Es ist kein Unternehmen der "New Social Economy«, sondern ging in seiner Geschichte durch viele Krisen und weist auch Schwächen auf. Bevor ich das Projekt zur Nachahmung weiterempfehle, bedarf es jedoch einiger vertiefender Analysen und Überlegungen. 